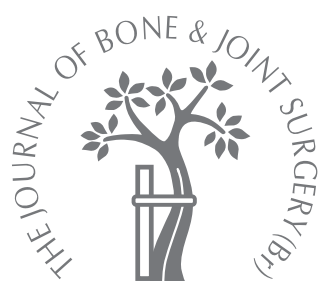

T. Matsubara,

K. Kusuzaki,

A. Matsumine,

H. Murata,

Y. Marunaka,

S. Hosogi,

A. Uchida,

A. Sudo

From Department of

Cell Molecular

Physiology, Kyoto

Prefectural

University Graduate

School of Medical

Science, Kyoto, Japan

T. Matsubara, MD,

Orthopaedic Surgeon

A. Matsumine, MD, Assistant

Professor

A. Uchida, MD, Dean

A. Sudo, MD, Professor

Department of Orthopaedic

Surgery

Mie University Graduate

School of Medicine, Edobash

2-174 Tsu City, Mie 514-8507،

Japan.

K. Kusuzaki, MD, Professor

- Y. Marunaka, MD, Professor

n. S. Hosogi, MD, Research

Fellow

Department of Molecular Cell

Physiology

= H. Murata, MD, Assistant

Professor

Department of Orthopaedic

Surgery

Kyota Prefectural University

Graduate School of Medical

Science, Kajii-cho 465

Kawaramachi-Hirokoji,

Kamigyo-ku Kyoto 602-8566,

Japan.

Correspondence should be sent to Professor K. Kusuzaki; email: kusuzaki@koto.kpu-

m.ac.jp

(C)2010 British Editorial Society of Bone and Joint Surgery doi:10.1302/0301-620X.92B6. $23788 \$ 2.00$

J Bone Joint Surg $[\mathrm{Br}]$ 2010;92-B:760-2.
- ASPECTS OF CURRENT MANAGEMENT \section{Photodynamic therapy with acridine orange in musculoskeletal sarcomas}

Limb salvage involving wide resection and reconstruction is now well established for managing musculoskeletal sarcomas. However, involvement of major nerves and vessels with a large volume of muscle and skin may result in a useless limb, contributing to depression and a low quality of life. We have been studying alternative treatments for musculoskeletal sarcoma since 1990 , and have recently established a regime using photodynamic surgery with cells labelled with acridine orange, photodynamic therapy with cells treated similarly and radiodynamic treatment using the effect of $X$-rays on such cells.

These techniques have been used after marginal or intra-lesional resection of tumours since 1999 and have enabled maintenance of excellent limb function in patients with sarcomas.

The use of photodynamic therapy (PDT) with haematoporphyrin or precursors such as porphyrin or aminoleuvelic acid has been shown to prevent local recurrence, when employed with minimally invasive surgery in early-stage superficial tumours of the skin, lung, oesophagus and bladder. The principle of this treatment is that a photosensitiser, such as haematoporphyrin, should first specifically or selectively accumulate in the tumour cells. Photon energy (hv) from a light source then excites the photosensitiser which activates intracytoplasmic oxygen. The activated oxygen oxidises fatty acids on the organelle (lysosomes membrane or DNA and RNA in the cell, resulting in death of the tumour cells due to necrosis or apoptosis. The targets of the photosensitisers differ. For example, haematoporphyrin groups bind to mitochondria.

As light beams, even from a laser system, cannot reach deeper than the skin or mucosa, sarcomas localised in deep areas are not suitable for PDT. However, irradiation of the tumour with a light beam is easily performed during surgery for resection of the tumour. If an incomplete intralesional procedure is performed accidentally, PDT might be useful in killing tumour cells which have leaked into the surgical area. We considered that if PDT could kill tumour cells, they might be eliminated without damage to normal tissues, preserving limb function without local recurrence.

Limb salvage with wide resection of the tumour followed by reconstruction using various types of endoprostheses, bone allograft or autograft in the treatment of musculoskeletal sarcomas has advanced markedly over the last 30 years. ${ }^{1-5}$ However, satisfactory recovery of function may not be achieved and most patients are still not able to run or swim fast, jump confidently, or throw a ball a long distance. ${ }^{6-8}$ These disabilities limit the activity of the patients. Better means of achieving satisfactory recovery of function after limb salvage are needed. We have therefore been investigating new photodynamic therapies since 1990. ${ }^{9-13}$ The use of haematoporphyrin may result in dermatitis induced by light and it requires a relatively long time to accumulate in tumour tissue. PDT with haematoporphyrin also requires the use of expensive laser beams. We therefore employed acridine orange $(\mathrm{AO})$ as a photosensitiser. This was extracted from coal tar in the late 19th century as a weak basic dye for staining clothes or microorganisms. Its biological properties include antitumour activity, ${ }^{14,15}$ photosensitising activity, ${ }^{16,17}$ and toxicity against bacteria, malarial parasites, and fungi. ${ }^{17-21}$ It has a low molecular weight of $463 \mathrm{u}$, and is reported to be capable of rapid flow into the cytoplasm through the cell membrane to bind to DNA, ${ }^{22} \mathrm{RNA}^{23}$ and lysosomes. $^{24}$ Many experimental studies have shown that $\mathrm{AO}$ has properties as a photosensitiser and is useful for PDT in the treatment of cancer. $^{25-29}$ However, the clinical application of $\mathrm{AO}$ in cancer therapy has not been described. This may be because of the potential 
toxic effects of $\mathrm{AO}$, which has been reported to induce mutagenic activity in bacteria. ${ }^{18,19}$ The carcinogenicity of $\mathrm{AO}$ has yet to be confirmed experimentally. ${ }^{30}$ An International Agency for Research on Cancer (IARC) report ${ }^{31}$ in 1978 classified AO into group 3, meaning that the agent is not currently classifiable in terms of carcinogenicity in humans. The local administration of $\mathrm{AO}$ to patients for screening for gastric and cervical cancer has been described, ${ }^{32}$ but none have developed new cancers induced by AO during the current follow-up of 11 years.

Our basic studies have shown that AO binds densely to lysosomes and acidic vesicles, emitting orange fluorescence after excitation by blue light in viable cultured mouse osteosarcoma cells and binds sparsely to RNA, emitting green fluorescence after excitation. $\mathrm{AO}$ has a unique feature in that the polymer type emits orange fluorescence, whereas the monomer shows green fluorescence under excitation by blue light. ${ }^{13}$ Osteosarcoma cells transplanted into mice emitted green fluorescence after intraperitoneal or intravenous injection of $\mathrm{AO}$ followed by blue light excitation, while normal muscle and adipose tissue cells did not. Hence, tumours could be visually localised under a fluorescence surgical microscope, through an effect of fluorovisualisation. ${ }^{12}$ We have confirmed that most human malignant bone and soft-tissue tumours are sensitive to staining with $\mathrm{AO}$, as specimens which have been resected emit intense green fluorescence after exposure to a solution of $\mathrm{AO}$ with blue light excitation. Although the mechanisms underlying selective binding of $\mathrm{AO}$ to musculoskeletal sarcoma are unclear, staining with $\mathrm{AO}$ is useful for visualising the tumour during surgery under fluorescence microscopy. We also found that $\mathrm{AO}$ had a strong cytocidal effect on mouse osteosarcoma cells after blue light excitation, both in vitro ${ }^{9}$ and in vivo, ${ }^{10}$ suggesting that it might be useful for PDT against musculoskeletal sarcoma. Since the concentration of AO solution $(1 \mu \mathrm{g} / \mathrm{ml})$ used in our clinical study was very low, and it was only administered locally, we believe that the risk of carcinogenesis induced by AO in patients may be significantly lower than that occurring from most other known anticancer agents.

When using porphyrin or its derivatives for $\mathrm{PDT}^{33}$ a laser beam with high energy focused over a narrow area is commonly used as the excitation light source. With $\mathrm{AO}$ we have used a high-power xenon lamp (SANEI Electric MIG Co., Ltd., Tokyo, Japan), since blue light illumination over a wide area is necessary for fluorovisualisation of $\mathrm{AO}$ and for the strong cytocidal effect of AO-PDT on the tumour cells spread widely throughout the surgical field during curettage. Xenon lamps are much cheaper than lasers. A recent study has shown that the cytocidal effect of AO-PDT is dependent not only on the wavelength (blue light, $466.5 \mathrm{~nm}$ ), but also on the lux $(\mathrm{lx})$ value of the light. Hence, while blue light needs to be used for microscopic curettage, full-wave light obtained from the xenon lamp without an interference filter is more effective than blue light alone for AO-PDT, because of the much higher lux of the lamp. We have therefore slightly modified our technique of AO-PDT during the last two years by using full-wave light.

Before the application of AO-PDT to human sarcomas, we carried out a simulation study of curettage supported by AO-PDT, using a mouse model. ${ }^{10}$ This showed that AOPDT after macroscopic and microscopic curettage of a mouse osteosarcoma significantly inhibited local recurrence of the tumour. The rate of recurrence in the control group was $80 \%$, compared with only $23 \%$ in the grouptreated using AO-PDT. We also observed that low-dose $\mathrm{X}$-ray irradiation of $5 \mathrm{~Gy}$ to mouse osteosarcoma after exposure to AO (radiodynamic therapy with acridine orange: AO-RDT) showed the same strong cytocidal effect as that of AO-PDT. ${ }^{11}$ Such irradiation has the advantage of reaching deeper areas of the human body compared with a light beam, although the deleterious effects on normal tissues are greater. The results of these basic studies suggest that AO-PDT and AO-RDT might be applicable for limb salvage in patients with malignant tumours of bone and soft tissue. If effective, patients might recover almost full limb function, with only a low risk of local recurrence.

We therefore conducted a clinical study to determine the feasibility and usefulness of AO-PDT with AO-RDT in human musculoskeletal sarcomas. We found an overall rate of recurrence of $10 \%$, almost the same as that after wide resection of the tumour. ${ }^{34-36}$ None of the five patients who had received AO-PDT with AO-RDT had recurrent tumour during a follow-up of more than two years. In the one case treated with AO-PDT alone the tumour recurred after 21 months, longer than would have been expected after macroscopic curettage of high-grade malignant sarcoma. Although the duration of follow-up was relatively short, we are convinced that the treatment employed was beneficial. The use of AO-PDT with, or without AO-RDT, inhibits local recurrence of musculoskeletal sarcomas, as most highgrade malignant sarcomas will recur within six months after intralesional excision. ${ }^{34-36}$ However, it is uncertain for how long this treatment might remain effective.

With the exception of one patient, the function of the limb was restored to the pre-operative level, and all patients were satisfied with the recovery of function. Compared with the results after wide resection of the tumour followed by limb reconstruction, recovery of function after AO-PDT (with or without AO-RDT) was superior in our study. We expect these patients to spend the remainder of their lives as normal, non-handicapped individuals. Local administration of AO, AO-PDT or AO-RDT was not associated with any complications, such as skin hypersensitivity to light, which is often encountered with PDT using porphyrin or its derivatives, ${ }^{33}$ and the patient does not need to avoid exposure to the sun even in the early phase after surgery.

AO-PDT with or without AO-RDT appears to be a promising new regime for the preservation of limb function in patients with a musculoskeletal sarcoma. This approach may also be applicable to other solid tumours, although larger studies with follow-up are required. 
No benefits in any form have been received or will be received from a commercial party related directly or indirectly to the subject of this article.

\section{References}

1. Kotz R, Dominkus $\mathbf{M}$, Zettl T, et al. Advances in bone tumour treatment in 30 years with respect to survival and limb salvage: a single institution experience. Int Orthop 2002;26:197-202.

2. Plötz W, Rechl H, Burgkart R, et al. Limb salvage with tumor endoprostheses for malignant tumors of the knee. Clin Orthop 2002;405:207-15.

3. Bickels J, Wittig JC, Kollender Y, et al. Distal femur resection with endoprosthetic reconstruction: a long-term followup study. Clin Orthop 2002;400:225-35.

4. Wunder JS, Leitch K, Griffin AM, Davis AM, Bell RS. Comparison of two methods of reconstruction for primary malignant tumors at the knee: a sequential cohort study. J Surg Oncol 2001;77:89-99.

5. RödI RW, Ozaki T, Hoffmann C, et al. Osteoarticular allograft in surgery for highgrade malignant tumours of bone. J Bone Joint Surg [Br] 2000;82-B:1006-10.

6. Lane JM, Christ GH, Khan SN, Backus SI. Rehabilitation for limb salvage patients: kinesiological parameters and psychological assessment. Cancer 2001:92:1013-19.

7. Davis AM, Sennik S, Griffin AM, et al. Predictors of functional outcomes following limb salvage surgery for lower-extremity soft tissue sarcoma. J Surg Oncol 2000;73:206-11.

8. Refaat Y, Gunnoe J, Hornicek FJ, Mankin HJ. Comparison of quality of life after amputation or limb salvage. Clin Orthop 2002;397:298-305.

9. Kusuzaki K, Minami G, Takeshita H, et al. Photodynamic inactivation with acridine orange on a multidrug-resistant mouse osteosarcoma cell line. Jpn J Cancer Res 2000;91:439-45

10. Kusuzaki K, Aomori K, Suginoshita T, et al. Total tumor cell elimination with minimum damage to normal tissues in musculoskeletal sarcomas following photodynamic therapy with acridine orange. Oncology 2000;59:174-80.

11. Hashiguchi S, Kusuzaki K, Murata $\mathbf{H}$, et al. Acridine orange excited by low-dose radiation has a strong cytocidal effect on mouse osteosarcoma. Oncology 2002;62:8593.

12. Kusuzaki K, Suginoshita T, Minami G, et al. Fluorovisualization effect of acridine orange on mouse osteosarcoma. Anticancer Res 2000;20:3019-24.

13. Kusuzaki K, Murata $\mathbf{H}$, Takeshita $\mathbf{H}$, et al. Intracellular binding sites of acridine orange in living osteosarcoma cells. Anticancer Res 2000;20:971-5.

14. Lewis MR, Goland PP. In vivo staining and retardation of tumors in mice by acridine compounds. Am J Med Sci 1948;215:282-9.

15. Korgaonkar KS, Sukhatankar JV. Anti-tumour activity of the fluorescent dye, acridine orange, on Yoshida sarcoma (ascites). Br J Cancer 1963;17:471-3.

16. Zdolsek JM, Olsson GM, Brunk UT. Photooxidative damage to lysosomes of cultured macrophages by acridine orange. Photochem Photobiol 1990;51:67-76.

17. Sastry KS, Gordon MP. The photodynamic inactivation of tobacco mosaic virus and its ribonucleic acid by acridine orange. Biochim Biophys Acta 1966;129:32-41.

18. Zampieri A, Greenberg J. Mutagenesis by acridine orange and proflavine in Escherichia coli strain S. Mutat Res 1965;2:552-6.
19. McCann J, Choi E, Yamasaki E, Ames BN. Detection of carcinogens and mutagens in the Salmonella/microsome test: assay of 300 chemicals. Proc Natl Acad Sci USA 1975:72:5135-9.

20. Giorgio A, Rambaldi M, Maccario P, Ambrosone L, Moles DA. Detection of microorganisms in clinical specimens using slides prestained with acridine orange (AOS). Microbiologica 1989;12:97-100.

21. Rickman LS, Oberst R, Long GW, et al. Rapid diagnosis of malaria by acridine orange staining of centrifuged parasites. Lancet 1989;1:68-71.

22. Kapuscinski J, Darzynkiewicz Z, Melamed MR. Interactions of acridine orange with nucleic acids: properties of complexes of acridine orange with single stranded ribonucleic acid. Biochem Pharmacol 1983;32:3679-94

23. Amagasa J. Mechanisms of photodynamic inactivation of acridine orange-sensitized transfer RNA: participation of singlet oxygen and base damage leading to inactivation. J Radiat Res (Tokyo) 1986;27:339-51.

24. Zelenin AV. Fluorescence microscopy of lysosomes and related structures in living cells. Nature 1966;212:425-6.

25. Tomson SH, Emmett EA, Fox SH. Photodestruction of mouse epithelial tumors after oral acridine orange and argon laser. Cancer Res 1974;34:3124-7.

26. Tatsuta M, Yamamura H, Yamamoto R, et al. Destruction of implanted gastric tumors in rats by acridine orange photoactivation with an argon laser. Eur $\mathbf{J}$ Cancer Clin Oncol 1984;20:543-52.

27. Prosser E, Cox D, O'Kennedy R, Carroll K, van der Putten W. Effects of coumarins, haematoporphyrins and acridine orange on the viability and growth of Landshütz ascites tumour cells, in the presence and absence of photoradiation. Cancer Lett 1990;52:71-7.

28. Ishikawa S, Nemoto R, Kanoh S, Kobayashi K, Ishizaka S. Photodynamic inactivation of bladder cancer cells (MGH-U1) sensitized with acridine orange and irradiated by argon laser. Tohoku J Exp Med 1984;144:265-71.

29. Zdolsek JM. Acridine orange-mediated photodamage to cultured cells. Apmis 1993;101:127-32.

30. Van Duuren BL, Sivak A, Katz C, Melchionne S. Tumorigenicity of acridine orange. Br J Cancer 1969;23:587-90.

31. No authors listed. Monographs on the evaluation of carcinogenic risks to humans. Vol. 16. 1978: 145. http://monographs.iarc.fr/ENG/Monographs/vol16/volume16.pdf (date last accessed 18 February 2010)

32. Kato A. Gastrofiberscopic diagnosis with acridine orange fluorescence. Gastroenterol Endosc 1970;12:351-62.

33. Vrouenraets MB, Visser GW, Snow GB, van Dongen GA. Basic principles, applications in oncology and improved selectivity of photodynamic therapy. Anticancer Res 2003;23:505-22.

34. Sondak VK, Chang AE. Clinical evaulation and treatment of soft tissue tumors. In: Weiss SW, Goldblum JR, eds. Enzinger and Weiss's soft tissue tumors. Fourth ed. St Louis: Mosby, 2001:28-32.

35. Zagars GK, Ballo MT, Pisters PW, et al. Prognostic factors for patients with localized soft-tissue sarcoma treated with conservation surgery and radiation therapy: an analysis of 1225 patients. Cancer 2003;97:2530-43.

36. Duffaud F, Digue L, Mercier C, et al. Recurrences following primary osteosarcoma in adolescents and adults previously treated with chemotherapy. Eur $\mathrm{J}$ Cancer 2003;39:2050-7. 